\title{
STRUCTURAL HEALTH MONITORING OF ROTATING BLADES ON HELICOPTERS
}

\author{
Aleksey MIRONOV ${ }^{1}$, Pavel DORONKIN ${ }^{1}$, Aleksander PRIKLONSKY ${ }^{1}$, \\ Igor KABASHKIN ${ }^{2}$ \\ ${ }^{1}$ D un D centrs, 7 Margrietas str. LV-1046 Riga, Latvia \\ ${ }^{2}$ Transport and Telecommunication Institute, 1 Lomonosova str., LV-1019 Riga, Latvia \\ E-mails: 1aleksei@ddcentrs.lv(correspondingauthor); kiv@tsi.lv
}

Received 26 April 2016; accepted 03 June 2016

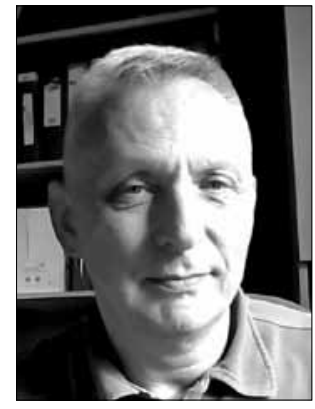

Aleksey MIRONOV, Dr sc. ing.

Date and place of birth: 1954, Tashkent, Uzbekistan.

Education: Riga Institute of Civil Aviation Engineering, Latvia.

Affiliation and functions: scientific director of D un D centrs since 2004 and Chairman of Board of Aviation Research Centre.

Research interests: vibration diagnostics of turbine engines, bearings, gears, structural health monitoring of aviation and transport structures.

Publications: over 70 scientific papers.

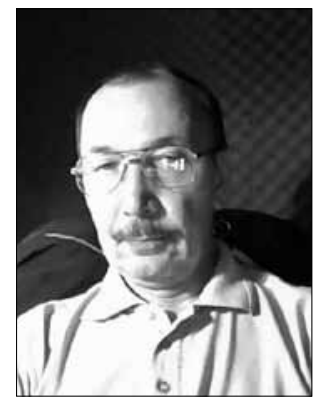

\section{Pavel DORONKIN, Engineer}

Date and place of birth: 1956, Almaty, Kazakhstan.

Education: Riga Institute of Civil Aviation Engineering, Latvia.

Affiliation and functions: Technical director of D un D centrs Board member of Vibroacoustic Laboratory.

Research interests: monitoring systems for aviation and civil structures applications, vibration and structural analysis, experimental testing equipment and software.

Publications: over 35 scientific papers.

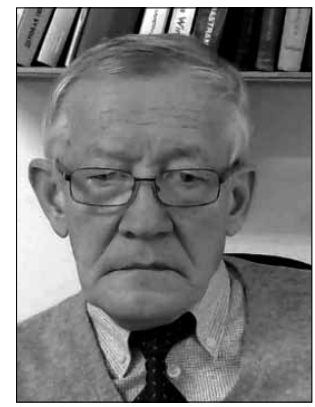

Alexander PRIKLONSKIY, Dr sc. ing.

Date and place of birth: 1948, Riga, Latvia.

Education: Riga Institute of Civil Aviation Engineering, Latvia.

Affiliation and functions: leading researcher of D un D centrs.

Research interests: vibration diagnostics of turbine engines, bearings, structural analysis and modelling of aviation and transport structures.

Publications: over 30 scientific papers.

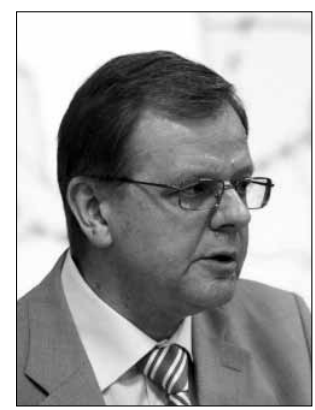

Igor KABASHKIN, Prof., Dr hab. sc. ing.

Date and place of birth: 1954, Riga, Latvia.

Education: Dr hab. sc. ing. degree in Engineering (1993, Riga Aviation University),

Dr sc. ing. degree in Aviation (1981, Moscow Aviation Institute), Diploma of Aviation

Radio Engineer (1977, Riga Civil Aviation Engineering Institute).

Affiliation and functions: Professor of Transport and Telecommunication Institute.

Research interests: transport, logistics, telematics, electronics, reliability and modelling of complex system.

Publications: more than 400 research papers and books, 68 patents. 
Abstract. The paper discusses the structural health monitoring of rotating blades on helicopters (RBH) based on the application of Modal Analysis. The study discussed in this paper includes the experimental validation of stateof-the-art techniques for the on-line measurement of dynamic signals of helicopter rotating units, optimization of the sensor type for rotating unit measurement, analysis of the practical applicability of modal analysis techniques for condition-based monitoring of rotating structures and estimation of the efficiency of the experimental system for the identification of practical damages of blades. The research was conducted using helicopter blade models operating within an experimental test bench. The capabilities of diagnostic technique application to main rotor gears and bearings are also presented. Conclusions are made about the successful analysis of the operational modal analysis technique applicability for the structural health monitoring of a rotating blade, and its effectiveness for damage identification. Two strategies of the RBH repair - with a continuously condition-based monitoring with the proposed technology and one without such monitoring, were discussed. The Markov chain reliability models for each strategy were analyzed and the reliability improvement factor for the proposed monitoring technology in comparison with a traditional one was evaluated. It is shown that the reliability improvement factor is more effective for the proposed method as compared to the traditional one.

Keywords: condition monitoring, rotating blades, vibration diagnostics, operational modal analysis, structural health monitoring.

\section{Introduction}

Improving the reliability of aviation in recent years has become a very urgent task. This problem becomes especially important for helicopters. This is because the dynamic loads on components in helicopters differ substantially from the similar loads on airplanes. This requires more attention to the maintenance of helicopters, which increases the maintenance and operation cost and the cost of their life cycle in total. One of the radical ways to improve the operational efficiency of aviation is the use of the Health and Usage Monitoring Systems. Structural Health Monitoring (SHM) seems capable of helping to reduce the maintenance and operational costs, which amounts to about 25 per cent of the direct operating cost of the helicopter (Sbarufatti et al. 2010). The widespread use of the SHM allows the use of Condition Based Maintenance (CBM), which can reduce the life cycle cost of helicopters without a significant change in their construction.

Condition monitoring based on vibration diagnostic techniques is applied widely for rotating machines while the SHM unites data acquisition and analysis in order to control the technical condition of structures throughout their life cycle (Hall 1999). The SHM defines an approach that provides the possibility to reveal dangerous changes of a construction resulting from damage caused or due to functionality failure (Kessler et al. 2002). Aviation has the highest potential return from the prevention of damages with the assistance of either the SHM or vibration diagnostics, as regular technical maintenance of airplanes results in as much as $27 \%$ of the cost of an aircraft's life cycle (Hall, Conquest 1999). Today, the main methodical tools of the SHM are the methods of modal analysis, including numerical and experimental techniques; however, these methods do not consider rotating structures.

Changes in the dynamic behavior of a structure, represented in the form of modal parameters, are the basis of a defect location strategy (Magpantay 2003; Mironov et al. 2015a). The evaluation of the modal parameters of an object is not an easy task for such operating structures as aviation constructions, especially rotating blades. Typically, test excitation is required to acquire the proper response for the modal parameters' computation. Experimental Modal Analysis techniques (EMA) consider such an activity. Aircraft or wind generator blades are not the objects to which this method is applicable. In flight or when driven by wind, the streaming airflow affects the rotating blades, loading it both statically and dynamically. Due to such circumstances the Operational Modal Analysis (OMA) is applied that uses only the response of the stressed structure excited by the surrounding environment for the evaluation of modal features. When considering heavy loaded rotating blades, this kind of influence is sufficient for the excitation of natural modes.

From multiple approaches the widest application was found for the approaches using the conversion in the time domain, like the Stochastic Subspace Identification (SSI) based methods, and in the frequency domain (Frequency Domain Decomposition (FDD).

Kiddy (Kiddy, Pines 2001) came the closest to solving the problem of the on-line monitoring of a rotating blade. Their Modal Analysis method developed for a non-rotatable structure was used for detecting the ballistic defect of a blade. The method was applied to two kinds of structures: a beam rotating in a vacuum and a non-rotatable blade of the Hughes TH-55A helicopter. The method proved capable of detecting the defects with a high modal power. At the same time, while detecting faults with a low modal power, the authors faced a number of problems. Luczak (Luczak et al. 2010) ran the static and dynamic testing of a full scale helicopter rotor blades using toe optical Fiber Bragg Grating and classical strain sensors as well as non-contact measurement techniques. The estimated modal models were compared with the 
experimental data in terms of natural frequency, damping ratio and mode shape. The comparison displayed that the accuracy of the results is frequency dependent and that the discrepancy between models grows in frequency. The use of Wireless Sensor Networks (WSN) for real time vibration monitoring promises to deliver a significant contribution to rotor performance monitoring and blade damage identification (Sanchez et al. 2013). From the application side, the monitoring system of a helicopter rotor requires diagnostic strategies for: a) rotor performance, b) blade integrity, and c) local damage monitoring. From the technological implementation side, the most relevant attributes for the WSN design have been identified as: (i) adequate distributed diagnostic algorithms for blade interrogation, (ii) efficient electronics for processing, sensing and harvesting, and (iii) suitable communication protocols for synchronized sensing.

This paper examines the application of the OMA methods in the HMS for the condition monitoring of rotor blades in flight or in operation (with wind generation), both excited by a streaming air flow.

The research study discussed in this paper aims:

- to experimentally validate state-of-the-art techniques for the on-line measurement of the dynamic signals of helicopter rotating units;

- to optimize the sensor type for the dynamic signal measurement of rotating units;

- to check the practical applicability of modal analysis techniques for the SHM of rotating structures;

- to study the efficiency of an experimental condition monitoring system for the identification of the practical damages of blades.

The key task of the study is to estimate thepractical applicability and efficiency of the EMA and OMA techniques for the SHM using the testing method. The more similar the behavior between typical mechanical parameters and the measured modal parameters in response to the test impact is, the higher the efficiency of the modal analysis technique. Typical mechanical parameters are represented by the stiffness and integrity of the blade models, but modal parameters are calculated as the eigenvectors of the natural modes of these models.

\section{Main assumptions and tasks}

Any structural defect related to the loss or redistribution of mass (erosion, chipping/flaking, deformation), stiffness (splits and cracks, changes of the physical and mechanical properties), and damping capacity (material ageing) causes changes in the dynamic properties of the structure. The above mentioned modal parameters reflect dynamic properties; therefore, any modification of the technical condition of the structure may be evaluated using its modal parameters. The method of modal analysis allows the experimental determination of the dynamic characteristics of a structure, including the frequencies, mode shapes and modal damping.

To achieve the main goals of the study the following tasks have been determined:

- development ofspecific samples of rotating blades for the structural modelling of their dynamic property modification;

- numerical modelling of the experimental blade model, and simulation of failures;

- motivation of the types and number of modes of which modal parameters shoud be used for the diagnosis;

- experimental study of the modal parameters of blade models in both standing and rotating conditions;

- experimental study of the modal parameters of modified blade models;

- estimation of modal parameter variation under the modification of a state.

In this study, the authors applied numerical modelling using the finite-element technique (FET) and then both the EMA and OMA techniques to study the modal parameters of the blade models. Shock test excitation and response measurement were used for the modal parameter estimation of standing blade models. Two different OMA techniques the (EFDD in frequency and CCI-CVA in time domains) were applied for the rotating blade modal estimation.

\section{Numerical modelling}

The numerical modelling of the blade model natural modes was discussed earlier (Mironov et al. 2015b). The main types of the natural mode shapes of a blade model were also considered. The lateral and longitudinal framing and skinning of the blade model is similar to that of a typical wing. The spar section of the blade represents an encasement formed by the back and front walls linked by ribs, forming the transverse framing and shaping the aerodynamic contour of the blade (Fig. 1).

The bending stiffness of the blade model is defined by both: the spar and three longitudinal beams

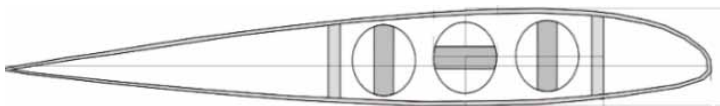

a)

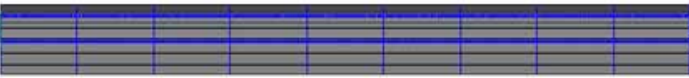

b)

Fig. 1. Blade model construction: a) plan view; b) cross-section 
(stiffeners), which have a rectangular profile and can be fixed in arbitrary positions. The vertical and horizontal moments of inertia of the stiffener profile relate as 10 to 1 . The variation of the stiffener orientation allows controlling the flexural stiffness of the blade model evenly along the full length. This way of the stiffness modification simulates the uniform stiffness loss of the composite blades in operation, which may happen due to the solar radiation. A preliminary modelling provided a study of typical natural blade mode shapes, their identification and the predicted modal parameter sensitivity to the global modification of the model parameters.

\section{Experimental study of the blade models}

\subsection{Blade models}

The experimental study of the blade models included the dynamic testing, modal analysis, identification, and comparison of the modal parameters of three blade models. The dynamic testing varied depending on the standing and rotating modes and was repeated for each modification of the model's mechanical properties. Depending on the testing mode (standing or rotating) the EMA or the OMA technique was applied to the data processing. Three blade models were manufactured, similar to the previously described numerical model. The natural blade model (Fig. 2) was made of plywood armed by three reinforced steel stiffeners having the ratio of orthogonal moments of inertia of 10 to 1 .

By changing the orientation of the stiffeners, it becomes possible to control the bending stiffness of the blade. In the first stage of the experimental study, the testing of the standing blade models at three grades of stiffness was considered:

- three stiffeners are set vertically (the maximum bending stiffness in the thrust direction);

- three stiffeners are horizontal (the lowest stiffness in thrust but the maximum in the rotation plane);

- two horizontal stiffeners and one vertical (intermediate version).

The experimental research study of the standing models was conducted using a multichannel acquisition system for simultaneous conditioning and measuring of the dynamic signals from the sensors. The Pulse
Labshop and ARTeMIS software platforms provided the data analysis from the sensors distributed on the models. The dynamic signals of the acceleration and deformation sensors were used as the output data of the oscillating blade model. The result of the data processing was obtained as the parameters of the shapes, frequencies and damping factors of the natural modes of the blade models within the frequency range of $0-800 \mathrm{~Hz}$.

The number and types of the blade model modes that had to be calculated from the experimental data was determined based on the numerical modelling and the blade model structure. Points for the measurement or excitation were distributed on the blade model surface in seven cross-sections and in three longitudinal axes (Fig. 2a), located along the leading edge (blue), along the spar (red) and on the tail edge (green). The points were marked on the surface of the blade model. Each impact or measurement point may have from one to three DOFs (Degree of Freedom). In case of vibration measurements, 3-axial accelerometers provide 63 DOF (Degree of Freedom). The sensors of dynamic deformations placed at the same points and oriented along the model provide 21 DOF. The DOF set-up provides the identification of the maximum 5-6 order longitudinal modes in the rotation plane and thrust direction as well as twist modes. Such a set of natural modes is considered sufficient for the modal state characterization of blade models.

\subsection{Tests and modal analysis of standing blade models}

\subsubsection{Experimental estimation of stiffness}

An objective estimation of the blade model stiffness and its modification was conducted using measurements of the models' deflection ${ }^{8}$. A stepwise loading of the blade model cantilevered on the test bench led to its deflection that was measured in the trust direction at various stiffener positions. When all three stiffeners are set in the vertical position, the model deflection is almost twice as little as at the lowest stiffness configuration (all stiffeners horizontally).

The stiffness increment between two ultimate stiffener positions was the metric of the stiffness modification. This parameter varies for the three hand-made

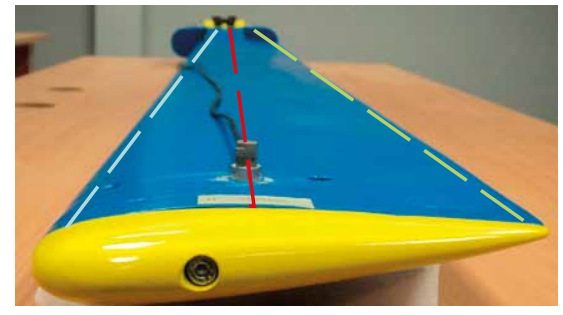

a)
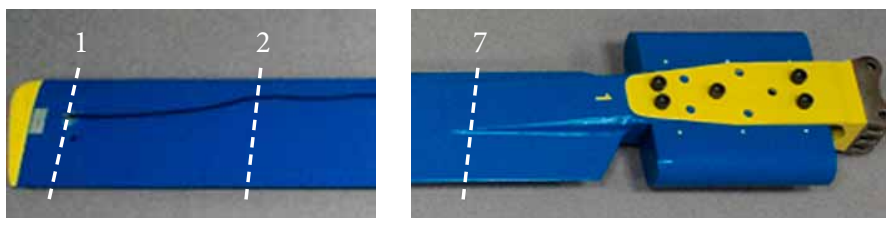

b)

Fig. 2. Natural blade model: a) tip view; b) plan view 
models $(1.79 \ldots 1.91)$ as an effect of their mechanical property scatter.

\subsubsection{MA of the standing blade models}

The EMA is the most useful for studying the dynamic properties of blade models in a standing mode. The "Roving hammer" technique considers the test impacts using a special hammer with a force transducer. Each impact point has one Degree of Freedom (DOF) oriented normally to the model surface. By a sequence of impacts the hammer "rounds" all DOF on the blade model surface, while the response of each impact is measured by two 3-axial transducers mounted on the blade tip and the root (Fig. 2). The data processing at this stage used two signal types: the excitation signal of the impact hammer and the response signals of both 3-axial accelerometers. The Frequency Response Functions (FRF) for each DOF were determined sequentially by a multiple repeat and data validation.

The Enhanced Frequency Domain Decomposition (EFDD) technique was applied for the modal analysis of the experimentally obtained data of the standing blade models. The available upper limit of the frequency range of modal identification depends on the measurement setup. With 7 cross-sections, where sensors are fixed, there is the opportunity to identify the $5^{\text {th }}$ or $6^{\text {th }}$ bending mode maximum. For the three tested blade models the maximum frequency of the $6^{\text {th }}$ mode does not exceed $500 \mathrm{~Hz}$. Within the upper bandwidth, fifteen natural modes were identified. These modes then were used for the comparative analysis of the modal properties in different test conditions. The identified modes include six vertical bending modes, four - horizontal bending, and five of them were twist modes. The FRF diagrams illustrating the FRF distribution (along and across the blade model) provide the validation of the EMA application results.

The EMA demonstrated the modal frequency decrease caused by the stiffness $\operatorname{loss}^{8}$; however, the estimation of the shape modification needs a special parameter. To estimate the modification of the $\mathrm{k}^{\text {th }}$ mode, the Modal Parameters Variation $\left(\mathrm{MPV}_{\mathrm{k}}\right)$ is used as an integrated parameter, which considers both the modal frequency and the shape modification from its initial state (or etalon). The group of modal parameters obtained experimentally using the modal analysis in the state $S$ of the blade model could be written as an eigenvector matrix

$$
M_{S}=\left[m_{i, j, k}\right]
$$

where $i$ - number of the DOF in one section $(i=1 \ldots 3)$; $j$ - number of sections $(j=1 \ldots 7) ; k$ - mode number $(1 \ldots 15) ; m_{i, j, k}$ - eigenvalue measured at the $\mathrm{DOF}_{\mathrm{i}, \mathrm{j}}$ of the $k^{\text {th }}$ mode.

For monitoring purposes the modal parameter $M_{S}$ of the current state $S$ is compared with the modal parameter $M_{E}$ of the initial state $E$ (or etalon) by the calculation of their difference in a relative scale:

$$
\Delta \mathrm{M}_{\mathrm{S}}=\mathrm{M}_{\mathrm{S}} / \mathrm{M}_{\mathrm{E}^{-}}
$$

Keeping in mind that the $M P V_{k}$ is the estimator of the $\mathrm{k}^{\text {th }}$ mode, we may present (2) in another way:

$$
\Delta \mathrm{M}_{\mathrm{S}}=\mathrm{MPV}_{\mathrm{S}}=\frac{1}{k} \sum_{1}^{k} M P V_{k} \text {. }
$$

Table 1 shows the experimental estimates of the MPV (based on the EMA) caused by the stiffness changes induced by the stiffeners' turn from the minimum to maximum stiffness orientation.

The parameter MPV evaluates the change of the modal parameters of each blade model between its ultimate stiffness states. Thus, for the $1^{\text {st }}$ blade model in comparison with a $79 \%$ increment estimated by a direct measurement, the integrated $\mathrm{MPV}_{\Sigma}$ (using both frequency and shape variation) proved to be almost the same $-78 \%$. It should be noted that although separate estimates of frequency and shape may show certain discrepancy, the integrated $\mathrm{MPV}_{\Sigma}$ deviates from the directly measured value slightly. For example, the $2^{\text {nd }}$ blade model has a frequency change of $70 \%$, the change of the mode shape is $125 \%$, but the integrated $\mathrm{MPV}_{\Sigma}$ is $95 \%$, which is close to the $93 \%$ according to the directly measured metric. The maximum difference between the $\mathrm{MPV}_{\Sigma}$ and the directly measured variation of the blade stiffness does not exceed 5\% (for blade model No. 3).

Table 1. Modal assessments (MA) of blade model stiffness change (in \%)

\begin{tabular}{llllll}
\hline \multirow{2}{*}{ No } & Parameters & \multicolumn{3}{c}{ Blade model No } & Averaged \\
\cline { 2 - 4 } 1 & 1 & 2 & 3 & \\
\hline $\begin{array}{l}\text { Stiffness ratio } \\
\text { (directly } \\
\text { measured) }\end{array}$ & 79 & 93 & 79 & 87 \\
\hline 2 & $\begin{array}{l}\text { Relative } \\
\text { frequency } \\
\text { change }\end{array}$ & 87 & 70 & 82 & 80 \\
\hline 3 & $\begin{array}{l}\text { MPVs by } \\
\text { shapes }\end{array}$ & 69 & 25 & 82 & 92 \\
\hline & $\begin{array}{l}\text { MPV } \\
\text { integrated } \\
\text { (frequency \& } \\
\text { shapes both) }\end{array}$ & 78 & 95 & 82 & 85 \\
\hline 5 & $\begin{array}{l}\text { MPV } \\
\text { (to directly } \\
\text { measured) }\end{array}$ & $-0.6 \%$ & $1.2 \%$ & $-4.2 \%$ & $-1.2 \%$ \\
\hline
\end{tabular}

Conspicuous is the fact that the average of the integrated $\mathrm{MPV}_{\Sigma}$ for all the three models appeared to be pretty close to the directly measured parameters, which allows to assume that the given approach is suitable not only for the monitoring of a certain blade during operation but also for the diagnostics of the same-type blades. 


\subsection{Tests and modal analysis of rotating blade models}

\subsubsection{Technical requirements to the rotating system}

To complete the principal tasks of the study, a set of technical requirements has to be provided. The operational conditions of the rotating blade models must be approximated to original ones, which means:

- rotation speed range from an idle to a nominal mode;

- common and circular pitch control;

- irregular airflow streaming the rotating blade models.

The measurement part of the system must ensure the measurement of dynamic signals on the rotating blades and further data transfer to the non-rotating part of the system, which requires:

- power supply to the rotating measurement system;

- sensor allocation on the blade models, considering the limited dimensions and the weight of sensors;

- signal measurement in a determined dynamic range (frequency and amplitude);

- a multichannel acquisition unit for the simultaneous signal conditioning, analogue-to-digital conversion and data preparation;

- on-line transfer of the prepared data to the non-rotating part of the system.

\subsubsection{The test bench and measurement setup}

The experimental testing system that was newly created includes mechanical and measurement parts that together are able to satisfy the above mentioned requirements. The mechanical part, the experimental test bench, includes a main gearbox, a main rotor with controlled blade models and auxiliary systems for the operation (Fig. 3a).

The $15 \mathrm{~kW}$ DC motor drives the rotor with the blade models through the main gearbox. Each experimental blade model has a similar layout of sensors'

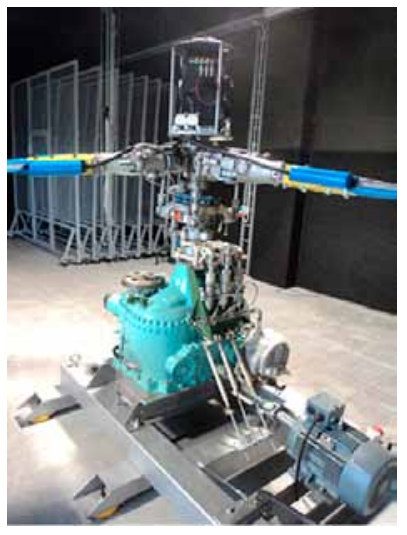

a)

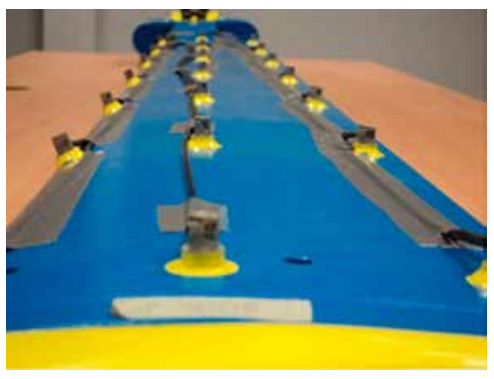

b) allocation that was defined in section 4.1 but differs in sensors and the measurement set up. Three-axial transducers with embedded preamplifiers are mounted on the surface of the blade model No 3 (Fig. 3b) using glued washers. Each transducer provides electric signals proportional to accelerations in the measurement point. Double integrated signals of acceleration in three directions form a $3 \mathrm{D}$ vector of displacement on the surface spot covered by a washer. Three DOF measured in one point provide a higher resolution; however, each transducer has a distinct mass that modifies the mechanical properties of the blade model and also interacts with the aerodynamic flow. The blade models No 1 and 2 (Fig. 3c) have a set of dynamic deformation transducers acting as strain gauges. An advanced piezoelectric film transducer (Fig. 4a) is insensitive to static deformations and measures dynamic deformations of the model surface.

Glued on the upper (low-pressure) surface of the blade model, this ultra-light sensor is exposed to stretching or compression together with the enveloped surface area. The preamplifier located next to the sensor on a flexible base transforms the time-variant electric charge generated by the sensor into alternate electric voltage. Ultra-light and thin deformation transducers do not affect the practically of the surveyed model towards mechanical and aerodynamic features and are much cheaper than accelerometers. However, such sensors measure the relative deformation of the occupied surface area (greater than the spot under the accelerometer washer) in one direction only, which reduces the potential resolution of the technique. Further discussion refers to the data obtained using these dynamic deformation sensors.

The measurement system of each blade model ends with two cables plugged into the acquisition unit mounted on the rotor hub (Fig. 4b). A flexible cable enclosure protects the sensor wires but practically does not affect the boundary conditions of the blade models. The outputs of the preamplifiers of both accelerometers and dynamic gauges are compatible, so different measurement systems of the blade models may be alternately plugged-in to the same acquisition unit. The 48-channel acquisition unit (Fig. 4 b) provides a simultaneous signal

Fig. 3. The test bench for the experimental system and the blade models

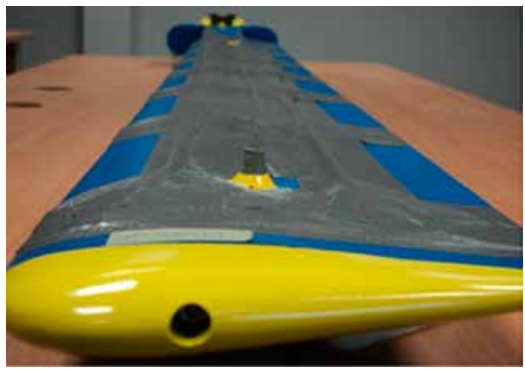

c) 


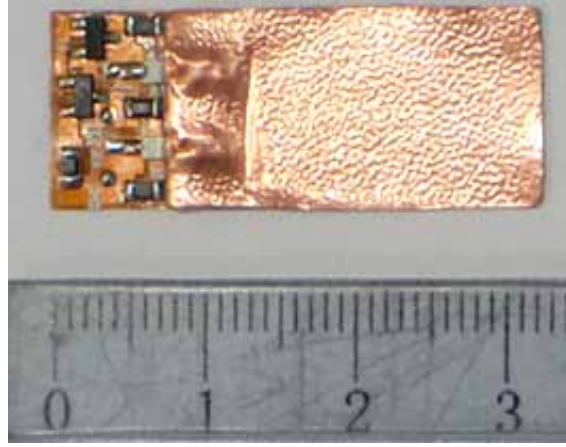

a)

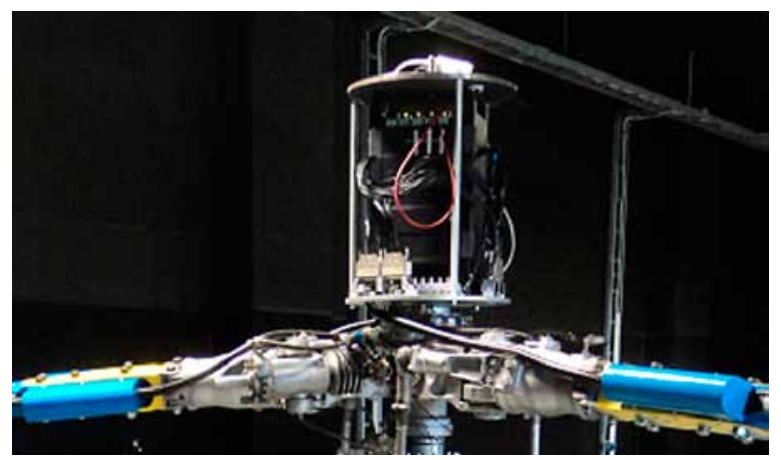

b)

Fig. 4. Single deformation transducer (a); acquisition unit on the rotor hub (b)

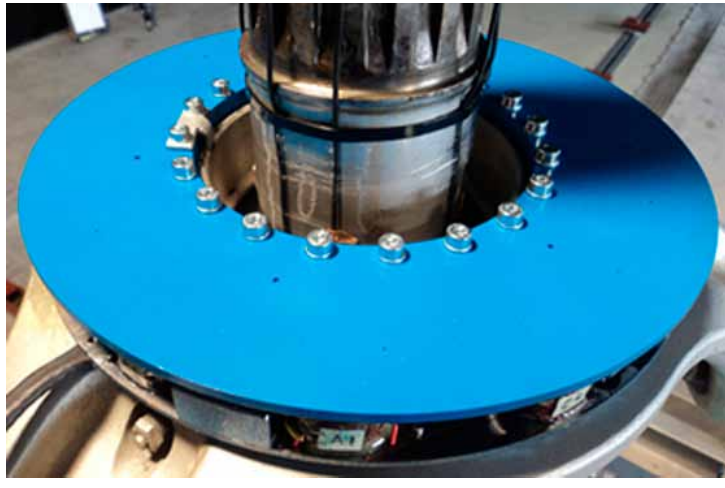

a)

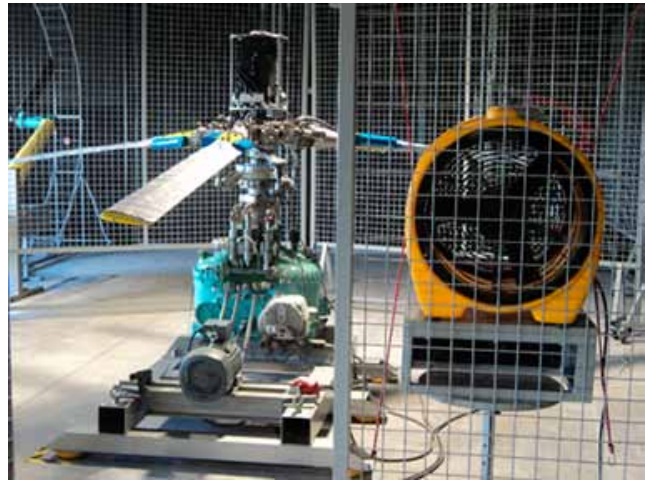

b)

Fig. 5. Photo of the electric generator of the harvesting system (a); the rotor test bench with the fan creating turbulent flow (b)

conditioning, the $\mathrm{AD}$ conversion and data preparation from the transducers of a single blade model as well as its transfer to a Wi-Fi router. The latter transfers the measured data wirelessly to an operator's PC which records data from all channels simultaneously and is at a safe distance from the operating rotor. The experimental harvesting system powered by a DC generator supplies the rotating measurement system, including the acquisition unit, preamplifiers of sensors and Wi-Fi router. The experimental DC generator is affiliated to the swash plate (Fig. 5a). Auxiliary systems of the test bench ensure the operation of the main gearbox, rotation speed and common/circular pitch of each blade. Apowerful fan could be used for a better simulation of natural conditions, for instance, air turbulence generation (Fig. 5b).

The operation test of the system proved that it conforms to the requirements specified above by the parameters outlined in Table 2.

Table 2. Parameters of the experimental system operation

\begin{tabular}{|c|c|c|}
\hline No & Subsystem & Parameters of operation \\
\hline 1 & $\begin{array}{l}\text { Mechanical system and electric } \\
\text { drive }\end{array}$ & Controllability from idle to nominal operation mode \\
\hline 2 & Measurement systems & $\begin{array}{l}\text { Blade } 1 \text { - } 21 \text { 3-axial accelerometers; } \\
\text { Blade } 2 \text { - } 42 \text { deformation sensors and two 3-axial accelerometers; } \\
\text { Blade } 3 \text { - } 49 \text { deformation sensors }\end{array}$ \\
\hline 3 & Data acquisition unit & 48 simultaneously operating measurement channels \\
\hline 4 & Wi-Fi data transmission & $\begin{array}{l}\text { Up to } 150 \mathrm{Mbit} / \mathrm{s} \text { providing real-time transmission from } 48 \text { simultaneously measured } \\
\text { channels }\end{array}$ \\
\hline 5 & Harvesting system & Power supply of the measurement system since $10 \%$ nominal rotation speed \\
\hline
\end{tabular}




\subsubsection{Verification of the applicability of OMA techniques in the rotating mode}

The OMA technique application refers to the dynamic actuation of the blade models and the measurement of their response. In the operational mode the static forces (centrifugal, aerodynamic drag and lift) load and the dynamic forces excite the rotating blade models as in a flight of an operational helicopter. The aerodynamic forces play the dominant role in the actuation of rotating blades; however, the periodic excitation from the main gear box also affects it. The response from the blade models is collected and measured by the rotating measurement system briefly described above. TheOMA application to the deformation signal measurement of blade model No 3 was carried out. Two OMA techniques were applied for the experimental data development: the Enhanced Frequency Domain Decomposition (EFDD) algorithm develops the measured data in the frequency domain, while the Stochastic Subspace Identification Canonical Variant Analysis (SSI-CVA) estimates the modal parameters directly from the raw measured time series.

The main task of the verification was to check the practical applicability of the OMA techniques for the condition monitoring of blade models dynamically loaded by airflow. The OMA technique is considered applicable if the computed MPV conforms to the experimentally measured stiffness increment. The software tool applying both EFDD and SSI-CVA algorithms to the measured data provides the set of eigenvectors describing the modal state of a blade model. As the measured dynamic signals are deformations, the extracted modal parameters of shape have a dimension of relative strain (\%). The number of identified modes determines the number of eigenvectors for the MPV computation. Only similar modes of comparable states are selected for the MPV parameter computation (see definition in section 4.2.2). The modification of the technical state of the blade is estimated by comparing the modal parameters of the current test to the blade reference state.

Firstly, the OMA abilities to identify the modes of a rotating blade were verified using a comparative analysis of the OMA extracted modal parameters with the standing blade ones. Practically all identified modes of the rotating blade match to the standing blade ones, taking into account the frequency shift caused by the static centrifugal and aerodynamic loads on a rotating blade. The resolution of the OMA techniques and the scatter of the obtained modal parameters were also studied. Applying the EFDD and SSI-CVA to the experimental data, the difference in the capability of the identification of these techniques was found. The first one allows a higher resolution for the blade modes of a lower order and shows less scatter between modal data in repeated tests. The second one identifies higher order modes better but
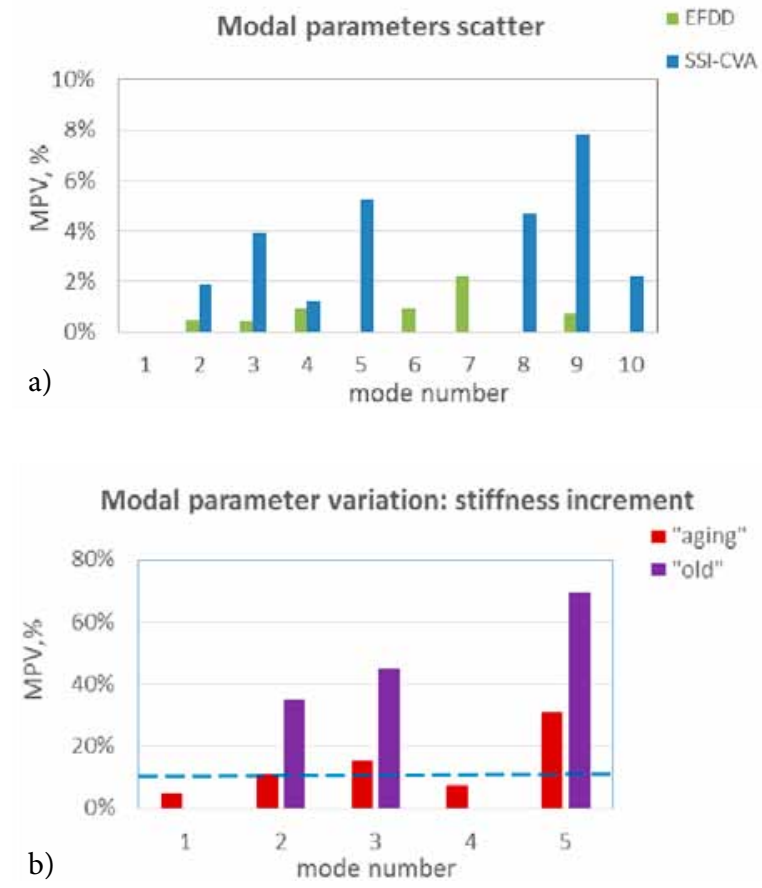

Fig. 6. Modal parameter variation: (a) - scatter between repeated states; (b) - gradual stiffness increment

the scatter of modal parameters is essentially greater. To estimate the scatter of the identified modal parameters, the blade was tested in the same state multiple times. Figure 6a illustrates scatter (relative difference between the values of the same parameter in the same technical state) of the integrated modal parameters of ten modes computed using both EFDD and SSI-CVA techniques. The modal shape parameters computed using EFDD scattered within $2 \%$, while the SSI-CVA provides more scatter (up to $8 \%$ ). Further practical application of both techniques would require to use them in parallel to increase the number of the identified modes and to improve their validation.

Therefore, the OMA techniques demonstrate an accurate identification of the modal parameters of rotating blade models if facilitated by proper measurement systems. The possibility of identifying modal parameters online in flight opens the gates to the condition monitoring of rotating blades using parameters, such as the MPV.

\subsection{Test damage identification}

Another task of the OMA technique verification was to study the efficiency of the experimental system for the identification of practical blade failures. Typically there are two types of rotating blade damages: global and local. The stiffness loss of the blade is an example of a global fault that occurs as the result of ageing, for instance under the sun radiation for composite blades. Contrary to global, different kinds of ballistic damages are typical local failures. They may result from small stones thrown up by rotating blades. 
Due to the specific construction of the blade models, it was possible to simulate the stiffness variation in a wide range. The modification of the position of all three stiffener from the horizontal to the vertical direction allows simulating the maximum stiffness increment of the blade model. For the blade model No 1 the maximum stiffness increment reached 79\% (Table 1) but for a smaller increment (one vertical stiffener) $-27 \%$. So, the ratio between two directly measured stiffness metrics is 2.93 . The on-line measurements of the $1^{\text {st }}$ blade dynamic deformations and the OMA application provide the modal parameters of this blade model in three configurations, including a reference and two gradually modified ones. Figure $6 \mathrm{~b}$ demonstrates the modal parameter variation of this blade in two states of stiffness ("aging" and "old") in relation to the reference state (3 horizontal stiffeners). The three bending modes in the trust direction (No 2, 3 and 5) are the most convenient for comparison. Other modes are less sensitive to such a technical state modification, for example, mode No 4 that is the $2^{\text {nd }}$ bending mode in the rotation plane. The average MPV for the maximum stiffness increment ("old") was 50\%, while for the "aging" case - only 17\%. Evidently, a 2.94 ratio between the MPV for both cases appears to be very close to a stiffness ratio of 2.93 measured experimentally. On this sample we may see that the MPV as a parameter may provide a quantitative estimation of the technical state modification for rotating blades.

For local damage imitation a $22 \mathrm{~mm}$ diameter through-hole was made that was located at the tip of the $1^{\text {st }}$ blade model out of the profile central part occupied by the stiffeners (Fig. 7a), so the blade's interior structure was not affected.
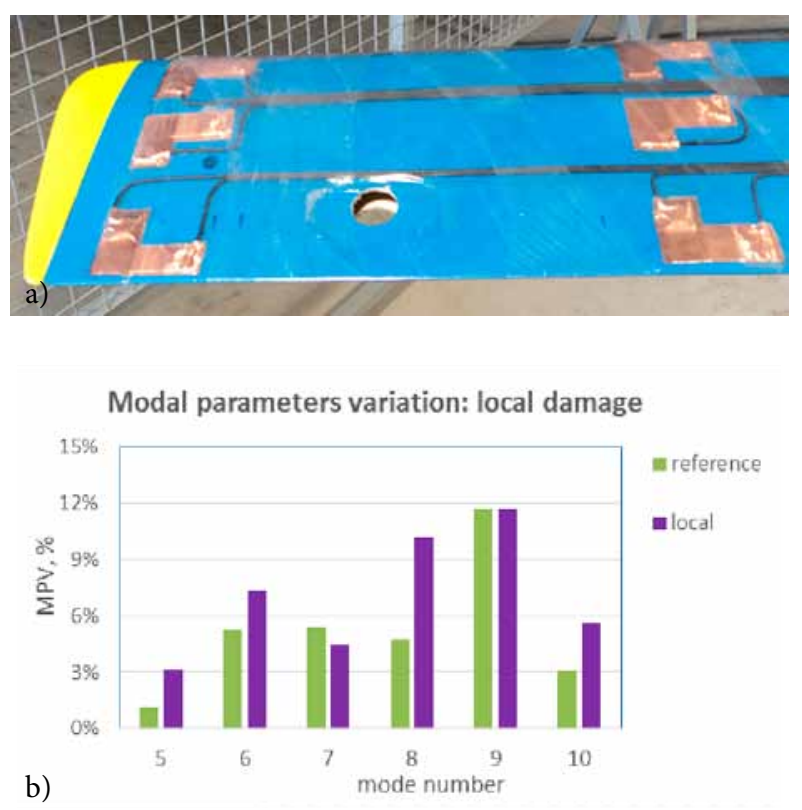

Fig. 7. Photo of the local damage of the blade model (a); local damage according to the modal parameter variation (b)

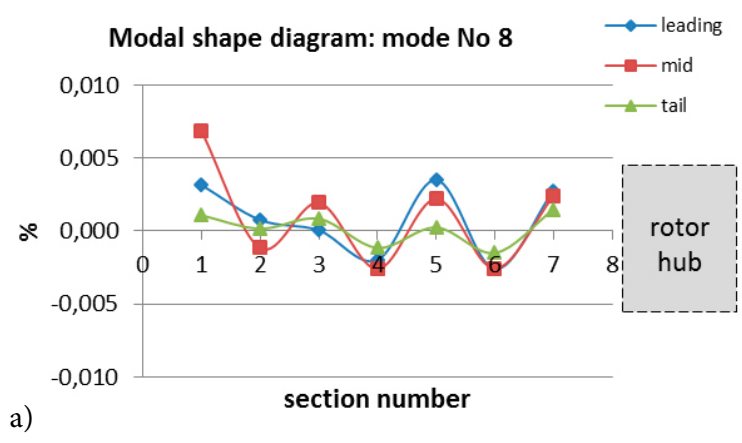

b)

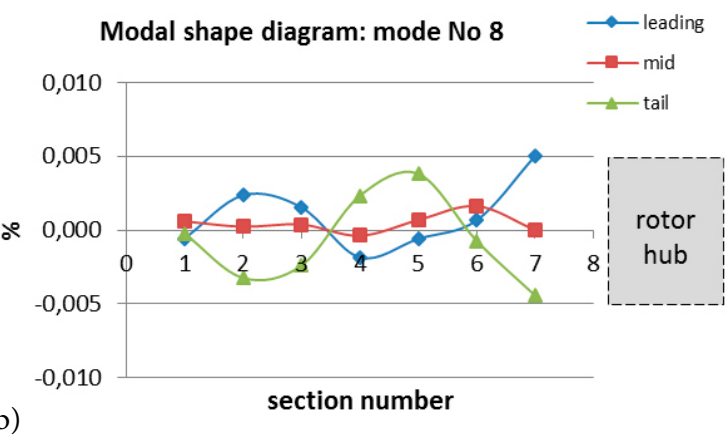

Fig. 8. Local damage identification: (a) - shape diagram of the $8^{\text {th }}$ mode; (b) - shape diagram of the $9^{\text {th }}$ mode

The stiffeners' configuration of the blade model remains unchangeable during this test. The through-hole damage affects locally unlike the global stiffness loss. That presupposes that the low order modes depending on global blade properties must remain unchanged; however, higher order modes may respond to the damage. Structural response is the subject of a mutual disposition of the local damage and the modal shape maximum. Figure $8 \mathrm{~b}$ illustrates the identification process of this specific damage based on the test series with and without local damage.

Two values of higher order modes' MPVs are compared for the two states: the scatter between the repeated tests of the reference state (green) and the MPV values of the locally damaged blade (purple). Evidently, some modes respond more to the fault while other do not "sense" it. The reason for the different response to local damage becomes understandable looking at the mode shape diagrams (Fig. 8). The $8^{\text {th }}$ mode (Fig. 8a) is a high order bending mode that has maximum deformations in the cross section of the damage location, which causes the maximum modification of this mode's parameters. The $9^{\text {th }}$ mode (Fig. 8b) is a $3^{\text {rd }}$ torque mode that has close to zero deformations at this section. In such a way even small local damages could be detected as well as the potential location could be "triangulated" by using some of the most sensitive modes.

To recap, the experimental study proves both the practical applicability of the OMA for rotating blades and its effectiveness for the damage identification. 


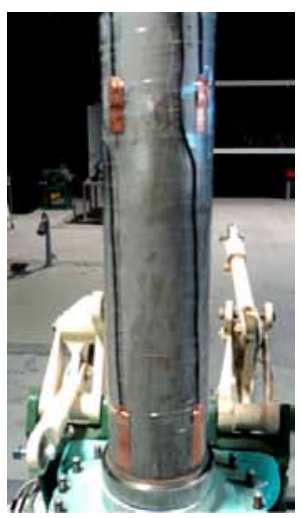

a)

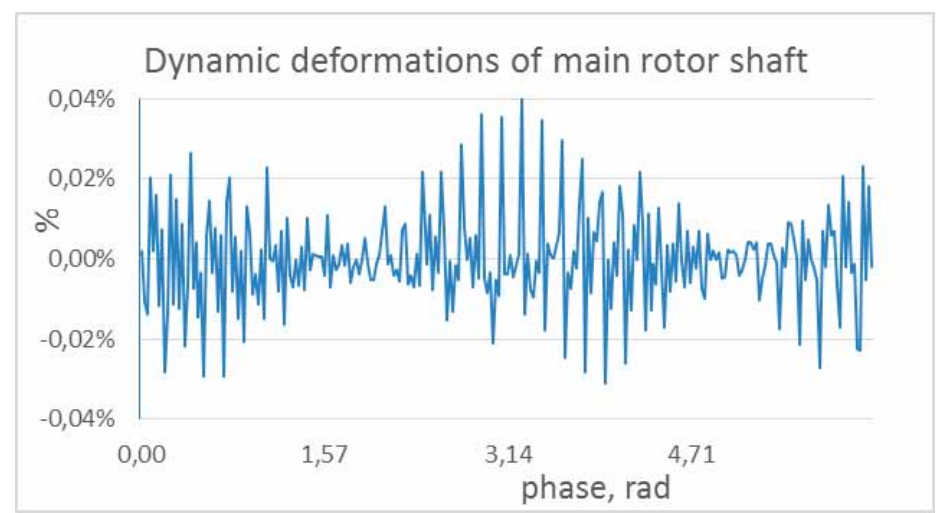

b)

Fig. 9. Deformation transducers on the main rotor shaft (a); the waveform of main rotor deformations in one revolution (b)

\subsection{Main rotor shaft monitoring}

The rotating system concurrently with the blade models may measure the deformations of the operating main rotor shaft using deformation transducers, as demonstrated in Figure 9.

The wide frequency band of the deformation transducers allows measuring the dynamic excitation and accordingly to condition monitoring of the main rotor shaft, the pinion driven by it as well as the inner rings of rotor bearings. The sample of the deformation signal measured and synchronously averaged in the revolution period of the rotor is presented in Figure 9b. The circled

\section{Circled track of main shaft deformation}
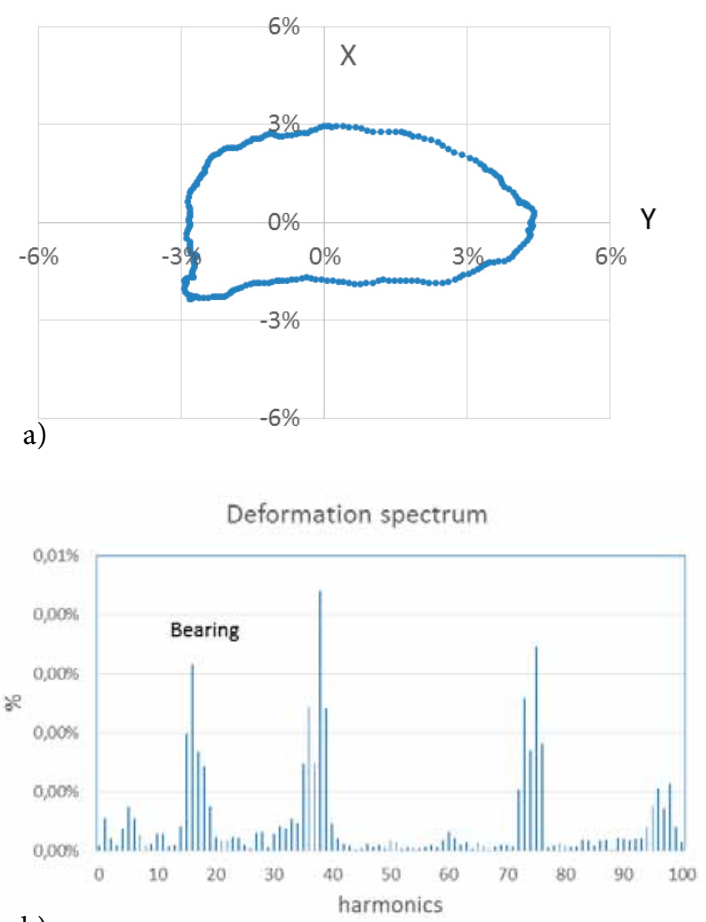

b)

Fig. 10. Data extracted from the shaft deformation signals: (a) - the circled track of the shaft deformation, (b) - the deformation waveform spectrum track of the main shaft deformation during the period of rotation (corresponding to an orbit) provides information about the rotor loading and the bearings' radial gap (Fig. 10a). The wide frequency band of the measured deformation signal may supply useful information about the technical condition of gears and bearings. The spectrum of synchronously averaged deformations (Fig. 10b) reflects the interaction of both pinion driven and the ball bearing that are the primary indicators of its technical state. Such useful information about the main rotor dynamic interactions creates the possibility for the diagnostic technique application to such inaccessible units as the main rotor gears and bearings, using deformations measured by the experimental system.

\section{Reliability of helicopter components with the OMA's application for condition monitoring}

The OMA technology proposed in this paper can be an effective instrument for the CBM. Let us investigate the impact of the adoption of technical condition diagnostics with the OMA technology on the reliability of helicopter components' (HC) operation.

The typical deterioration of functional capabilities of helicopter components is shown in Figure 11 (Campbell, Reyes-Picknell 2006). A condition-based monitoring (CBM) of parameter $\pi$ is carried out using the proposed technology with a sensitivity level $\alpha$. The moment $t_{\alpha}$ is the point in time when an incipient failure is first detected depends on the condition monitoring technique. If maintenance actions are not executed, the system failure occurs at time $T_{0}$ due to the development of degradation processes.

The following assumptions were made in the mathematical formulation:

- the service life of a $\mathrm{HC}$ is infinite;

- the HC monitoring and maintenance system should be able to find any defects in the unhealthy $\mathrm{HC}$ under monitoring before any failure happens; 


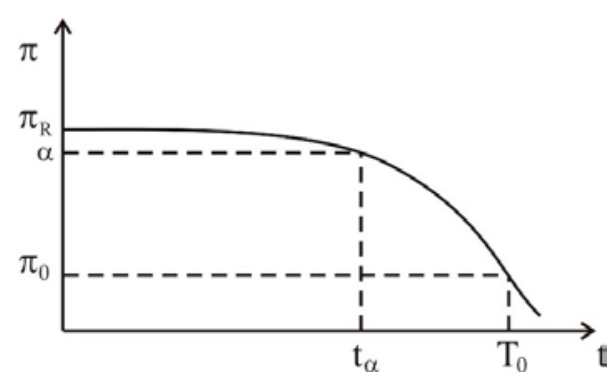

Fig. 11. Deterioration of functional capabilities

- the degradation rate for different modes of failure is constant with parameter $\lambda_{i}$, where $i=1, . . n$ is the number of detected modes of failures;

- in the case of operation without the CBM, the imode failure is hidden and continues to develop before the occurence of a system failure with the failure rate $\varphi_{i}, i=1, \ldots n$;

- when a failure is detected during condition-based monitoring with rate $\alpha_{i} \lambda_{m}$, a preventive maintenance is carried out with the repair rate $\mu_{i}$, where $i=1, . . n$ is nthe umber of detected modes of failures;

- the HC after maintenance actions becomes as good as new;

- the occurrence of critical failures and their repair have an exponential distribution with parameters $\lambda_{0}$ and $\mu_{0}$, respectively.

Let us compare two strategies of a $\mathrm{HC}$ repair - with a continuously condition-based monitoring with the proposed technology and without such monitoring in accordance of approach proposed in (Kabashkin 2010). For this purpose, we use the Markov chain model with a discrete random process whose future states only rely on their current states and are independent of their past states.

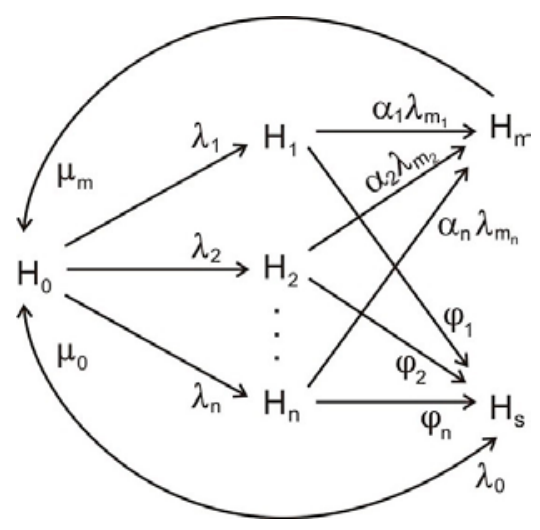

Fig. 13. Markov's state transition diagram for a system without a condition-based monitoring

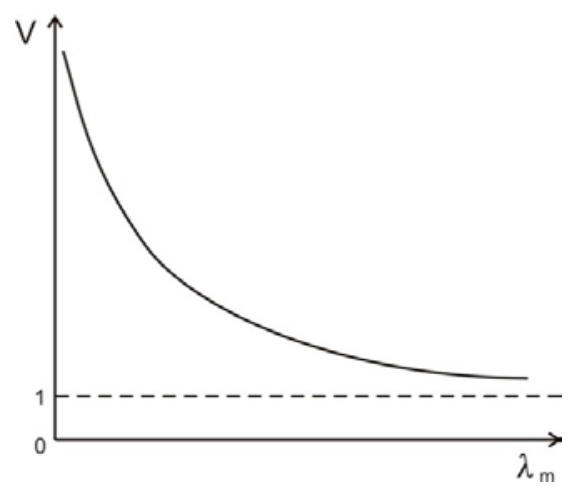

Fig. 12. Variation of the reliability deterioration coefficient

Model 1. Strategy of the HC repair without a condition-based monitoring.

The behavior of the examined system is described by the state transitions (Fig. 13): $H_{0}$ indicates state in which the system is operating and available for use; $H_{i}$ the appearance of an $\mathrm{i}$-mode failure $(i=1, \ldots n) ; H_{s}$ - the appearance of a system failure; $H_{m}$ - maintenance operations on the HC.

On the basis of the state transition diagram for Markov's process, shown in Figure 13, we can write the following system of Chapman-Kolmogorov's equations:

$$
\begin{aligned}
& P_{0}^{\prime}(t)=-\sum_{i=1}^{n} \lambda_{i} P_{0}(t)+\mu_{m} P_{m}(t)+\mu_{s} P_{s}(t) \\
& P_{i}^{\prime}(t)=\lambda_{i} P_{0}(t)-\left(\alpha_{i} \lambda_{m}+\varphi_{i}\right), \quad i=\overline{1, n} \\
& P_{m}^{\prime}(t)=\sum_{i=1}^{n} \alpha_{i} \lambda_{m} P_{i}(t)-\mu_{m} P_{m}(t) \\
& P_{s}^{\prime}(t)=\lambda_{s} P_{0}(t)+\sum_{i=1}^{n} \varphi_{i} P_{i}(t)-\mu_{s} P_{s}(t)
\end{aligned}
$$

The normalizing condition is

$$
P_{s}(t)+P_{m}(t)+\sum_{i=0}^{n} P_{i}(t)=1
$$

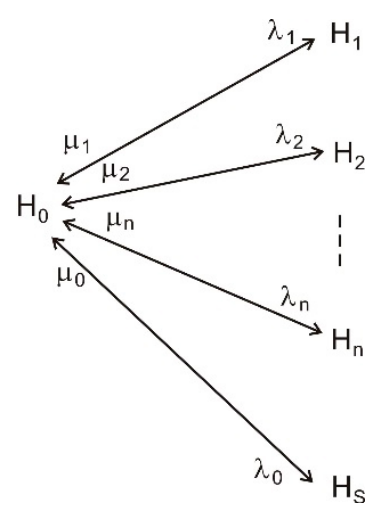

Fig. 14. Markov's state transition diagram for A system with a continuously condition-based monitoring 
After the transformation of the above mentioned set of equations, we can obtain the value for $P_{i}(i=1$, $\ldots n, m, s)$ via $P_{0}$ :

$$
\begin{aligned}
& P_{i}=\frac{\lambda_{i}}{\alpha_{i} \lambda_{m}+\varphi_{i}} P_{0}, i=\overline{1, n} ; \\
& P_{m}=\frac{\lambda_{m}}{\mu_{m}} \sum_{i=1}^{n} \frac{\alpha_{i} \lambda_{i}}{\alpha_{i} \lambda_{m}+\varphi_{i}} P_{0} ; \\
& P_{s}=\left(\frac{\lambda_{s}}{\mu_{s}}+\frac{1}{\mu_{s}} \sum_{i=1}^{n} \frac{\lambda_{i} \varphi_{i}}{\alpha_{i} \lambda_{m}+\varphi_{i}}\right) P_{0} .
\end{aligned}
$$

The value of $P_{0}$ can be obtained by a replacement $P_{i}$ $(i=1 \ldots n, m, s)$ in the normalizing equation:

$$
P_{0}=\left[\begin{array}{l}
1+\sum_{i=1}^{n} \frac{\lambda_{i}}{\alpha_{i} \lambda_{m}+\varphi_{i}}+\frac{\lambda_{m}}{\mu_{m}} \sum_{i=1}^{n} \frac{\alpha_{i} \lambda_{i}}{\alpha_{i} \lambda_{m}+\varphi_{i}}+ \\
\frac{\lambda_{s}}{\mu_{s}}+\frac{1}{\mu_{s}} \sum_{i=1}^{n} \frac{\lambda_{i} \varphi_{i}}{\alpha_{i} \lambda_{m}+\varphi_{i}}
\end{array}\right]^{-1} .
$$

The availability of the system for this model is $A=P_{0}$.

The numerical values associated with the calculation of the availability are often awkward for highly reliable systems. For this reason, it is more convenient to use the complement measure of availability, namely, unavailability U. Unavailability is the probability that an item will not operate correctly at a given time and under the specified conditions (Dunn 2002). It is opposite to availability.

$$
\begin{aligned}
& U=1-A=P_{s}+P_{m}+\sum_{i=1}^{n} P_{i} ; \\
& U=\frac{a_{1}}{1+a_{1}} ; \\
& a_{1}=\frac{\lambda_{s}}{\mu_{s}}+\sum_{i=1}^{n} \frac{\lambda_{i}\left(\mu_{s} \mu_{m}+\alpha_{i} \mu_{s} \lambda_{m}+\mu_{m} \varphi_{i}\right)}{\mu_{m} \mu_{s}\left(\alpha_{i} \lambda_{m}+\varphi_{i}\right)} .
\end{aligned}
$$

Model 2. Strategy of a HC repair with a continuously condition-based monitoring.

The behavior of the examined system is described by the state transitions (Fig. 14): $H_{0}$ indicates a state in which the system is operating and available for use; $H_{i}-$ the appearance of an $i$-mode failure $(i=1, \ldots, n)$ which is detected during condition-based monitoring, a preventive maintenance is started; $H_{s}$ - the appearance of a system failure.

On the basis of the state transition diagram for Markov's process, shown in Figure 14, we can write the following system of Chapman-Kolmogorov's equations:

$$
\begin{aligned}
& P_{0}^{\prime}(t)=\sum_{i=1}^{n} \mu_{i} P_{i}(t)+\mu_{0} P_{s}(t)-\lambda_{0} P_{0}(t) \\
& P_{i}^{\prime}(t)=\lambda_{i} P_{0}(t)-\mu_{i} P_{i}(t), \quad i=\overline{1, n} \\
& P_{s}^{\prime}(t)=\lambda_{0} P_{0}(t)-\mu_{0} P_{s}(t) .
\end{aligned}
$$

The normalizing condition is:

$$
P_{s}(t)+\sum_{i=0}^{n} P_{i}(t)=1 \text {. }
$$

After the transformation of the above mentioned set of equations, we can obtain the value for $P_{i}(i=0, \ldots n, s)$ :

$$
\begin{aligned}
& P_{i}=\frac{\lambda_{i}}{\mu_{i}} P_{0}, i=\overline{1, n} ; \\
& P_{s}=\frac{\lambda_{0}}{\mu_{0}} P_{0} ; \\
& P_{0}=\left(1+\sum_{i=0}^{n} \frac{\lambda_{i}}{\mu_{i}}\right)^{-1} .
\end{aligned}
$$

The unavailability of the system for this model is:

$$
\begin{aligned}
& U=1-A=P_{s}+\sum_{i=1}^{n} P_{i} ; \\
& U=\frac{a_{2}}{1+a_{2}} ; \\
& a_{2}=\sum_{i=0}^{n} \frac{\lambda_{i}}{\mu_{i}} .
\end{aligned}
$$

It is possible to evaluate the increase of the reliability of the $\mathrm{HC}$ with the continuously condition-based monitoring in comparison with the $\mathrm{HC}$ without such diagnostics with the help of the reliability improvement factor $V=U_{1} / U_{2}$, where $U_{1}$ indicates the unavailability of the system in the first model and $U_{2}$ - the unavailability of the system in the second one, in accordance with equations (4) and (5).

The analysis of the $V$ coefficient indicates that under the conditions $\mu_{s}=\mu_{m}=\mu_{0}=\mu$ and $\lambda_{i} \ll \mu_{\mathrm{r}}$ for all $\mathrm{i}$, which is done for the most highly reliable systems, its value can be described by a approximate expression $V=1+n \mu / \lambda_{m}$. In the Figure 12 the reliability improvement factor $V$ is shown as a function of value $\lambda_{m}$.

\section{Conclusions}

The research study presented in this paper provides the grounds for the application of operational modal analysis techniques for the condition monitoring of rotating blades in-flight. This finding is based on experimental tests of the principal technical aspects for the solution of this task, including:

- advanced deformation transducers describing the dynamic behavior of the operating blade,

- rotating data acquisition and wireless transmitting system measuring, and data transfer to a remote standing concentrator,

- an experimental harvesting system providing the continuity of power supply for the rotating measurement system. 
The most advanced EFDD and SSI-CVA techniques of the OMA have proved its applicability for the identification of the modal parameters of blades in a rotating mode. Both technical solutions and diagnostic techniques analysed the described experimental work demonstrated the functioning of the new approach to condition monitoring of the helicopter main rotor, including the blades and other units in-flight. Most of the obtained results are also applicable to the rotors of wind generators.

Two strategies of the HC repair - with a continuously condition-based monitoring with the proposed technology and without such a monitoring, were evaluated from the reliability point of view. It is shown that the reliability improvement factor for the proposed method shows that it is more effective in comparison with the traditional one.

\section{Acknowledgements}

The paper uses material related to the research study no. 1.32 project of the project "Establishment of Transport Mechanical Engineering Competence Center" in cooperation with the Investment and Development Agency of Latvia (L-KC-11-0002).

\section{References}

Campbell, J.; Reyes-Picknell, J. 2006. Uptime strategies for excellence in maintenance management. New York: Productivity Press. 357 p.

Dunn, W. 2002. Practical design of safety-critical computer systems. Reliability Press. 360 p.

Hall, S. R. 1999. The effective management and use of structural health data, in Proceedings of the 2nd International Workshop on Structural Health Monitoring. 8-10 September 1999, Stanford, CA, 265-275 p.

Hall, S.; Conquest, T. 1999. The total data integrity initiative - Structural Health Monitoring, the next generation, in Proceedings of the USAF ASIP Conference, 31 November 1999, San Antonio, Texas.

Kabashkin, I. 2010. Optimal monitoring strategies, in Wiley encyclopedia of operations research and management science. New York: John Wiley \& Sons, Inc. 6408 p.

Kessler, S.; Spearing, M.; Atalla, M., et al. 2002 Damage detection in composite materials using frequency response methods, Composites Part B:Engineering 33(1): 87-95. http://dx.doi.org/10.1016/S1359-8368(01)00050-6

Kiddy, J.; Pines, D. 2001. Experimental validation of a damage detection technique for helicopter main rotor blades, Journal of Systems and Control Engineering 215: 209-221. http://dx.doi.org/10.1243/0959651011540996

Luczak, M.; Peeters, B.; Dziedziech, K. 2010. Static and dynamic testing of the full scale helicopter rotor blades, in Proceedings of ISMA2010 including USD2010, 20-22 September, Leuven, Belgium, 2131-2144.

Magpantay, H. 2003. Modal identification from ambient vibration measurement: a technology for optimization of the performance of civil engineering structures. Ateneo de Naga University.
Mironov, A.; Doronkin, P.; Priklonsky, A., et al. 2015a. Condition monitoring of operating pipelines with operational modal analysis application, Transport and Telecommunication 16(4): 305-319.

http://dx.doi.org/10.1515/ttj-2015-0028

Mironov, A.; Doronkin, P.; Priklonsky, A., et al. 2015b. Effectiveness of application of modal analysis for the monitoring of stressed or operated structures, Aviation 19(3): 112-122. http://dx.doi.org/10.3846/16487788.2015.1104860

Sanchez, R.; Das, K.; Loendersloot, R., et al. 2013. Wireless sensor network for helicopter rotor blade vibration monitoring: requirements definition and technological aspects, in the 10th International Conference on Damage Assessment of Structures, DAMAS, 8-10 July 2013, Dublin, Ireland, 775-782.

Sbarufatti, C.; Manes, A.; Giglio, M., et al. 2010. Application of structural health monitoring over a critical helicopter fuselage component [online], [cited 2 February 2016]. Available from Internet: http://hdl.handle.net/11311/574961 